\title{
A X-ray diffraction analysis on graphene layers of Assam coal
}

\author{
BINOY K SAIKIA, ${ }^{\mathrm{a}, \mathrm{b} *}{ }^{\mathrm{R} A J A N I} \mathrm{~K}_{\text {BORUAH }}^{\mathrm{a}}$ and PRADIP $\mathrm{K} \mathrm{GOGOI}^{\mathrm{c}}$ \\ ${ }^{a}$ Analytical Chemistry Division, North East Institute of Sciences \& Technology (CSIR), Jorhat 785006 \\ ${ }^{b}$ Department of Chemical Sciences, Tezpur University, Napaam, Tezpur 784028 \\ ${ }^{\mathrm{c}}$ Department of Chemistry, Dibrugarh University, Dibrugarh 786004 \\ e-mail: binoyrrl@yahoo.com
}

MS received 20 June 2007; revised 30 October 2007

\begin{abstract}
The so-called turbostatic structure of carbons in coal with randomly oriented stacking of the lamellae (graphene) produces intense peaks, which are the dominant features in its X-ray diffraction profiles. The diffractogram may be conveniently divided into two regions of reciprocal space, the medium $\mathrm{S}$ region $(1<\mathrm{S}<3 \AA)$ and a high $\mathrm{S}$ region $(\mathrm{S}>3 \AA)$ where $S=4 \pi \lambda^{-1} \sin \theta$. To better understand the molecular level structure of high sulphur Assam coal, two coal samples (Tirap-1 and Tirap-2) from Tirap colliery of Makum coalfield, Assam (India) has been interpreted in this study by using the X-ray diffraction profiles. Random layered (graphene) structural parameters of these coals were determined by using X-ray diffraction technique, which showed that the $L_{a}$ and $L_{c}$ are $64.99 \AA$ and $22.63 \AA$ for Tirap- 2 and $55.54 \AA$ and $23.80 \AA$ for that of Tirap-1 coals respectively. The position of $\gamma$ band was found to be at $4 \cdot 34 \AA$ and $4 \cdot 13 \AA$ for Tirap-2 and Tirap-1 coals respectively. The number of layers and average number of carbon atoms (N) per aromatic graphene were found to be 21 and 8 for both the coal samples. Proximate, ultimate and ash analysis of the two coal samples were also carried out in this investigation.
\end{abstract}

Keywords. Coal structure; graphene layers; Assam coal.

\section{Introduction}

Coal is a natural carbonaceous material. The physicochemical properties of coal vary with its locations. The large varieties of organic and inorganic materials involved in the formation of coal makes them highly heterogeneous, both in physical and chemical structure. Structural characterization of coal represents one of the most important activities in coal science owing to its utilizations. The technological use of the factors such as structure, mineral matter associated with coal is of more practical importance because it creates new opportunities both for widening this raw material in coke making and conversion of coal in order to obtain liquid fuel as well as coal base chemical products.

The tertiary coals of Assam are of generally very low ash and high sulphur in nature. Besides their normal use as fuels, they are ideally suited for direct hydrogenation for production of synthetic oil. Although, mostly high in sulphur, the highly caking

*For correspondence low ash coals of this region can be profitably used even to some extent as blends in metallurgical coke making.

\section{$1.1 \quad X$-ray diffraction from coal}

The X-ray diffractogram of coal can be divided into three distinct segments, each of which is dominated by a specific type of scattering from the organic matrix of the coal. The intense segment are in the high-angle region, which is due to the scattering from small condensed aromatic layer ${ }^{1-2}$ and in the intermediate angle, which includes the intensity band caused by the packing of the layers of condensed aromatic units. Thus, X-ray diffraction has been one of the few orthodox methods used to estimate the size of the aromatic lamellae in coal, the average distance between lamellae and the mean bond distance.

For sufficiently large and strain free crystallites in polycrystalline specimen diffraction thereby predicts that the lines of the powder pattern will be exceedingly sharp. The diffuse and abnormally weak lines can be explained by a structure in which layers stacked in hexagonal close packing alternate in a random manner with larger stacked in cubic close 
packing. On the other hand, two-dimensional lattice lines are characterized by terminating sharply on the low angle sides but falling off gradually in intensity on the high angle sides. The two-dimensional lattices, i.e. random layer lattice, produce such type of reflections.

A broad intense carbon-related scattering peak with a ' $d$ ' value from 4 to $5 \AA$ has been indexed as the $\gamma$-band. It may be mentioned that the origin of this peak has not been successfully explained. It may be due to a packing effect of aliphatic side chains to alicyclic structures with parallel stacking although details are not studied until recently. ${ }^{3,4}$ But some other workers ${ }^{1,5}$ indicate that it may be due to a short-range order of aromatic lamellae, which has been distributed by side groups.

\subsection{Present study}

The aim of the present study is to determine some of the X-ray crystalline structural parameters of two coal samples from Makum coalfield in Tinsukia district of Assam. India. The parameters include the interlayer spacing and crystalline size along c-axis $\left(L_{c}\right)$ and in the layer sheet direction $\left(L_{a}\right)$, the average number of carbon atoms $(\mathrm{N})$ per aromatic lamellae, packing of the aromatic lamellae and the gamma band $(\gamma)$, which has been established as the structural parameters for evaluating the stacking (graphene layers) structure of crystalline carbon materials. ${ }^{6}$

\section{Methods and materials}

The coal samples were collected from Tirap colliery of Makum coalfield (latitudes $27^{\circ} 13^{\prime}-27^{\circ} 23^{\prime} \mathrm{N}$ and longtitutes $95^{\circ} 35^{\prime}-96^{\circ} 00^{\prime} \mathrm{E}$ ) Assam and ground to -200 mesh (BSS) size. Diffraction data were obtained using computer controlled X-ray diffractometre type XPART PRO (PHILIPS). The proximate analysis of the coal sample was done by standard methods. (IS: 1350 (Part I-1984). The elements C, H, and N were determined by the elemental analyzer, Perkin Elmer, model 2400. The total sulphur was determined by Sulphur Determinator (Leco, SC132) and the percentage of oxygen was calculated by difference. The forms of sulphur were determined by following standard methods (ASTM D 2492). Table 1 summarized these characterizations. The results show that it is having $3.20 \mathrm{wt} \%$ and $2.75 \mathrm{wt} \%$ sulphur along with $75.39 \mathrm{wt} \%$ and $75.90 \mathrm{wt} \%$ carbon for Tirap-2 and Tirap-1 coals respectively.

\section{Results and discussions}

\subsection{Crystalline parameters of the coals: Aromatic lamellae and crystallite size $\left(L_{c}\right.$ or $\left.L_{002}\right)$}

From the X-ray diffraction profiles (figure 1) of Tirap-2 and Tirap- 1 coal samples the X-ray crystalline parameter are determined semi-quantitatively and are shown in tables 2 and 3.

The mean crystallite size of powder composed of relatively perfect crystalline particle can be determined with the familiar Scherrer equation ${ }^{7}$ (neglecting the lattice strain),

$$
L_{h k l}=k \lambda / \beta_{0} \cos \theta,
$$

where $L_{h k l}$ is the mean dimension of the crystallite perpendicular to the plane $(h k l) ; \beta_{0}$ is the integral breadth or breadth at half maximum intensity of the pure reflection profile in radians; $k$ is a constant that is commonly assigned a value of unity.

For diffuse two-dimensional reflection, if axis ' $c$ ' is perpendicular to and axes ' $a$ ' and ' $b$ ' lie on the plane of the layer, the crystalline reflection will be of the type '001' and two-dimension reflection will be

Table 1. Physico-chemical properties of the coal samples (wt $\%$ as received basis).

\begin{tabular}{lcc}
\hline Parameters & Tirap-2 & Tirap-1 \\
\hline Proximate analysis & & \\
$\quad$ Moisture & 1.8 & 2.40 \\
Ash & 6.6 & 5.5 \\
Volatile matter & 45.4 & 44.9 \\
Fixed carbon & 46.2 & 47.2 \\
Forms of sulphur & & \\
Total sulphur & 3.20 & 2.75 \\
Pyritic sulphur & 0.64 & 0.26 \\
Sulphate sulphur & 0.09 & 0.23 \\
Organic sulphur & 2.47 & 2.26 \\
Ultimate analysis & & \\
C & 75.39 & 75.90 \\
H & 7.2 & 4.51 \\
N & 1.00 & 1.27 \\
O (by difference) & 16.41 & 18.32 \\
\hline
\end{tabular}

Table 2. X-ray structural parameters of coals from (10) band $\left(L_{a}\right)$.

\begin{tabular}{lcccc}
\hline Coals & $\theta$, Plane ' 10, & Value of $d$ & \\
$(\AA)$ & $L_{10}\left(L_{a}\right) \AA$ & $\gamma$ Band $\AA$ \\
\hline Tirap-2 & 24.52 & 1.79 & 64.99 & 4.04 \\
Tirap-1 & 23.13 & 1.96 & 55.54 & 4.13 \\
\hline
\end{tabular}


Table 3. X-ray structural parameters of coals from (002) band $\left(L_{c}\right)$.

\begin{tabular}{lccccc}
\hline Samples & $\theta$, Plane '002' & Value of $d(\AA)$ & $L_{002}\left(L_{c}\right) \AA$ & No of layers & $\begin{array}{c}\text { No of atoms } \\
\text { per layer }\end{array}$ \\
\hline Tirap-2 & 13.49 & 3.30 & 22.63 & 8 & 21 \\
Tirap-1 & 12.82 & 3.68 & $23 \cdot 80$ & 8 & 21 \\
\hline
\end{tabular}

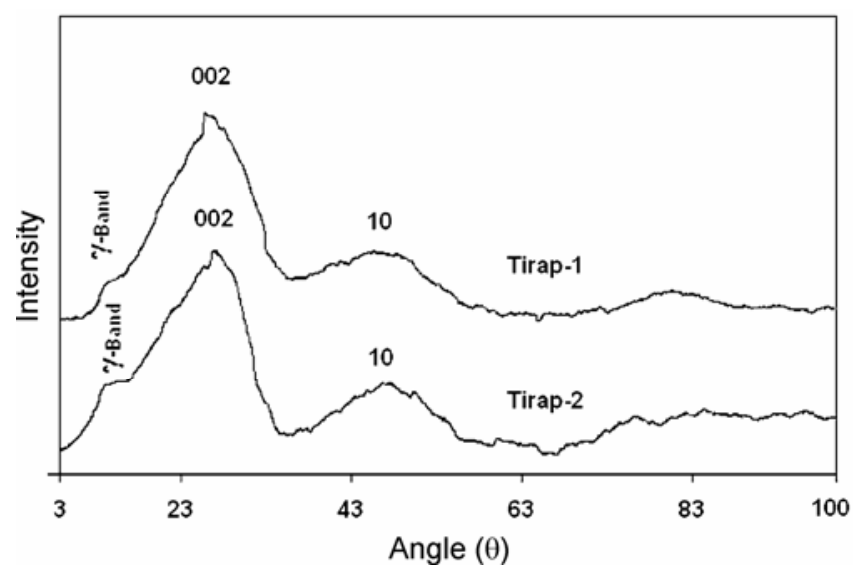

Figure 1. X-ray diffractogram of Tirap-1 and Tirap-2 coal samples.

of the index type ' $h k$ '. No general ' $h k l$ ' reflection will be observed. The size of two-dimensional layer in the plane of layer can be calculated from the observed breadth of ' $h k$ ' reflection, using the Scherrer equation $^{7,8}$ with an appropriate value of shape factor $k$. The breadth of the reflection on the $2 \theta$ scale at half intensity is,

$$
\beta_{1 / 2}=2 \lambda / L_{h k l} \cos \theta \text {. }
$$

Here, $L_{h k l}$ is the lamellae diameter of the twodimensional layer in a direction perpendicular to the diameter of the two-dimensional diffracting lattice ' $h k$ ' and $k=2$. $^{7}$ The crystallite size $\left(L_{c}\right)$ was found to be $22.63 \AA$ and $23.80 \AA$ for Tirap-2 and Tirap-1 coals respectively. It may however be mentioned that the result is in good agreement with that of earlier report. ${ }^{8}$ The histogram of vitrain shows that about $90 \%$ of carbon the size distribution of aromatic clusters is relatively narrow. However, above $90 \% \mathrm{C}$, the size distribution becomes significantly broader. The innermost atoms of edge groups are included to some extent in the layer when layer sizes are calculated. ${ }^{9}$ It is also based on the assumption that the layers are perfect. It may be noted that the amorphous portion refer to the structural elements of the coal which are not associated with the hexagonal network characteristics of the aromatic with the ad- joining side groups. This contributing factor decreases with increasing rank and runs completely parallel to the non-aromatic fraction. However, the evaluation of the non-aromatic portion of the coal structure from the amorphous portion requires an accurate knowledge of the influence of different factors such as foreign elements and non-aromatic structure on the amorphous fraction. It is worthwhile to mention that a qualitative study of exinite, vitrinite and micrinites led to the conclusion ${ }^{1,5}$ that for exinite, the average lamella is slightly smaller than that of corresponding vitrinites where as in the micrinite the aromatic cluster are distinctly larger.

$L_{a}$ or $L_{h k}$ may be determined to denote the mean dimension in the plane of a two-dimensional layer structure. This average lamellae diameter was determined by application of relation between lines width and lamellae diameter for the ' 10 ' peaks. From the observed half-maximum breadth of ' 10 ' plane, the mean layer dimensions are found to be $64.99 \AA$ and $55.54 \AA$ for the Tirap- 2 and Tirap-1 coals respectively.

\subsection{Determination of number of carbon atom $(N)$, packing aromatic lamellae and the average lamellae diameter $(L)$}

The average number of carbon atoms $(\mathrm{N})$ per aromatic lamellae can be calculated from the equation given else where ${ }^{8}$,

$$
N=0.32 L^{2} \text {. }
$$

The average number of carbon atoms was found to be 21 for both the coals. However, it may be mentioned that the method has been thoroughly tested using amorphous polymers having known size of aromatic clusters and followed an excellent basic agreement between the theory and experiment. ${ }^{10}$

\subsection{Packing of aromatic lamellae}

It is worthwhile to note that the parallel arrangement of the aromatic lamellae produces the so-called '001' 
reflection of which the most intense is ' 002 '. The average interlayer distance of the lamellae can be computed from the position of the '002' reflection, where as the average dimension of the packets of the lamellae in the direction perpendicular to their planes may be estimated from its half width

If each parallel layer consists of $\mathrm{N}$ layers, $\mathrm{L}_{002}$ for a parallel layer group is defined as ${ }^{7}$

$$
L_{002}=(N-1) d_{002} \text {, }
$$

or

$$
N=\left(L_{002}+d_{002}\right) / d_{002} .
$$

The number of layer as determined by this method using $L_{002}$ was found to be 8 for both the coals. In vitrinite, the ' 002 ' position progressively changes towards higher angles with increasing rank, and at the same time the band becomes sharper. ${ }^{10}$ Micrinites, as compared with the corresponding vitrinites, show a more perfect short-range order for the lamellae, and this leads to sharper and more intense ' 002 ' reflection. Thus, the interlayer distances as observed from this study were $3.30 \AA$ and $3.68 \AA$ for Tirap-2 and Tirap- 1 coals respectively. It is to be mentioned that the interlayer distance decreases with varying rank and their petrographic components of coals. ${ }^{10}$ Taking into accounts the 'turbostratic' structure for coals, the minimum separation between aromatic lamellae (graphenes) would be 3.30-3.68 $\AA$ for these coals. ${ }^{11}$

\subsection{Gamma band}

Some coals show a band intense scattering peaks with a $d$ value from 4 to $5 \AA$, which has been indexed as the gamma band. This gamma band is observed at $4.04 \AA$ and $4.13 \AA$ for Tirap- 2 and Tirap- 1 coal samples. The origin of the peaks has not yet been explained successfully. It may be due to a packing effect of aliphatic side chains to alicyclic structures with parallel stacking as indicated by some workers. But some authors indicated that it might be due to a short- range order of aromatic lamellae, which has been distributed by side groups.

\section{Conclusions}

Coal samples collected from the Tirap colliery of Assam, India showed the presence of graphene layers with the mean lamellae diameter values for $L_{a}$ and $L_{c}$ are 64.99 and $22.63 \AA$ for Tirap- 2 coal and 55.54 and $23.80 \AA$ for that of Tirap-1 coals respectively. The position of $\gamma$ band was found to be at $4.04 \AA$ and $4.13 \AA$ for Tirap- 2 and Tirap-1 coals respectively. The average number of carbon atoms $(\mathrm{N})$ per aromatic graphene (layer) and the number of layers were found to be 21 and 8 for both the Tirap- 2 and Tirap-1 coals respectively.

\section{Acknowledgements}

The authors are grateful to the Director, North-East Institute of Science and Technology (NEIST), Jorhat for giving permission to publish this paper. The author (BKS) is thankful to Mr B P Baruah, Scientist, NEIST, Jorhat for his suggestions. Authors are also thankful to the anonymous reviewers for valuable suggestions in improving the manuscript.

\section{References}

1. Cartz L, Diamond R and Hirsch P B 1956 Nature 117 500

2. Wertz D L 1995 Fuel 741431

3. Watanable I, Sakanishi K and Mochida I 2002 Energy and Fuel 1618

4. Mochida I and Sakanishi K 2000 Fuel 79221

5. Ruland W 1959 Acta. Crystallogr. 12679

6. Iwashita N and Inagaki M 1993 Carbon 311107

7. Klug P H and Alexander L E 1974 X-ray diffraction procedures (New York: John Wiley \& Sons)

8. Lowry H H (ed.) 1963 Chemistry of coal utilizations (New York: John Wiley \& Sons)

9. Diamond R Acta Crystallogr. 195811129

10. Nelson J B 1954 Fuel 33153

11. Hirsch P B 1954 Proc. Roy. Soc. (London) A226 143 